\title{
Concept and Definition of Complexity
}

\author{
Russell K. Standish \\ Mathematics and Statistics, UNSW
}

October 23, 2018

\section{Keywords}

Complexity, Emergence, Complex Systems, Information Theory, Graph Theory, Occam's Razor

\begin{abstract}
The term complexity is used informally both as a quality and as a quantity. As a quality, complexity has something to do with our ability to understand a system or object — we understand simple systems, but not complex ones. On another level, complexity is used as a quantity, when we talk about something being more complicated than another.

In this chapter, we explore the formalisation of both meanings of complexity, which happened during the latter half of the twentieth century.
\end{abstract}

\section{Introduction: Is complexity a quality or a quantity?}

The term Complexity has two distinct usages, which may be categorised simply as either a quality or a quantity. We often speak of complex systems as being a particular class of systems that are difficult to study using traditional analytic techniques. We have in mind that biological organisms and ecosystems are complex, yet systems like a pendulum, or a lever are simple. Complexity as a quality is therefore what makes the systems complex.

However, we may also speak of complexity as a quantity — with statements like a human being being more complex than a nematode worm, for example. Under such usage, complex and simple systems form a continuum, characterised by the chosen complexity measure.

Bruce Edmonds (1999) performed a comprehensive survey of complexity measures as part of his $\mathrm{PhD}$ thesis, however it has not been updated to include measures proposed since that time. However, it remains the most comprehensive resource of complexity measures available to date.

The aim of this chapter is not to provide a catalogue of complexity measures, but rather to select key measures and show how they interrelate with each other within an overarching information theoretic framework.

\section{Complexity as a quantity}

We have an intuitive notion of complexity as a quantity; we often speak of something being more or less complex than something else. However, capturing what we mean by complexity in a formal way has proved far more difficult, than other more familiar quantities we use, such as length, area and mass.

In these more conventional cases, the quantities in question prove to be decomposable in a linear way, ie a $5 \mathrm{~cm}$ length can be broken into 5 equal parts $1 \mathrm{~cm}$ long; and they can also be directly compared - a mass can be compared with a standard mass by comparing the weights of the two objects on a balance. 
However, complexity is not like that. Cutting an object in half does not leave you with two objects having half the complexity overall. Nor can you easily compare the complexity of two objects, say an apple and an orange, in the same way you can compare their masses.

The fact that complexity includes a component due to the interactions between subsystems rapidly leads to a combinatorial explosion in the computational difficulty of using complexity measures that take this into account. Therefore, the earliest attempts at deriving a measure simply added up the complexities of the subsystems, ignoring the component due to interactions between the subsystems.

The simplest such measure is the number of parts definition. A car is more complex than a bicycle, because it contains more parts. However, a pile of sand contains an enormous number of parts (each grain of sand), yet it is not so complex since each grain of sand is conceptually the same, and the order of the grains in the pile is not important. Another definition used is the number of distinct parts, which partially circumvents this problem. The problem with this idea is that a shopping list and a Shakespearian play will end up having the same complexity, since it is constructed from the same set of parts (the 26 letters of the alphabet - assuming the shopping list includes items like zucchini, wax and quince, of course). An even bigger problem is to define precisely what one means by "part". This is an example of the context dependence of complexity, which we'll explore further later.

Bonner and McShea have used these (organism size, number of cell types) and other proxy complexity measures to analyse complexity trends in evolution (Bonner, 1988, McShea, 1996). They argue that all these measures trend in the same way when figures are available for the same organism, hence are indicative of an underlying organism complexity value. This approach is of most value when analysing trends within a single phylogenetic line, such as the diversification of trilobytes.

\section{Graph Theoretic Measures of Complexity}

Since the pile of sand case indicates complexity is not simply the number of components making up a system, the relationships between components clearly contribute to the overall complexity. One can start by caricaturing the system as a graph - replacing the components by abstract vertices or nodes and relationships between nodes by abstract edges or arcs.

Graph theory (Diestel, 2005) was founded by Euler in the 18th century to solve the famous Königsberg bridge problem. However, until the 1950s, only simple graphs that could be analysed in toto were considered. Erdös and Renyi (1959) introduced the concept of a random graph, which allowed one to treat large complex graphs statistically. Graphs of various sorts were readily recognised in nature, from food webs, personal or business contacts, sexual relations and the Internet amongst others. However, it soon became apparent that natural networks often had different statistical properties than general random graphs. Watts and Strogatz (1998) introduced the small world model, which has sparked a flurry of activity in recent years to measure networks such as the Internet, networks of collaborations between scientific authors and food webs in ecosystems (Albert and Barabási, 2002).

Graph theory provides a number of measures that can stand in for complexity. The simplest of these is the connectivity of a graph, namely the number of edges connecting vertices of the graph. A fully connected graph, however, is no more complex than one that is completely unconnected. As connectivity increases from zero, a percolation threshold is reached where the graph changes from being mostly discontinuous to mostly continuous. The most complex systems tend to lie close to the percolation threshold. Another graph measure used is cyclomatic number of a graph, basically the number of independent loops it contains. The justification for using cyclomatic number as a measure of complexity is that feedback loops introduce nonlinearities in the system's behaviour, that produce complex behaviour.

Related to the concept of cyclomatic number is the number of spanning trees of the graph. A spanning tree is a subset of the graph that visits all nodes but has no loops (ie is a tree). A graph made up from several disconnected parts has no spanning tree. A tree has exactly one spanning 


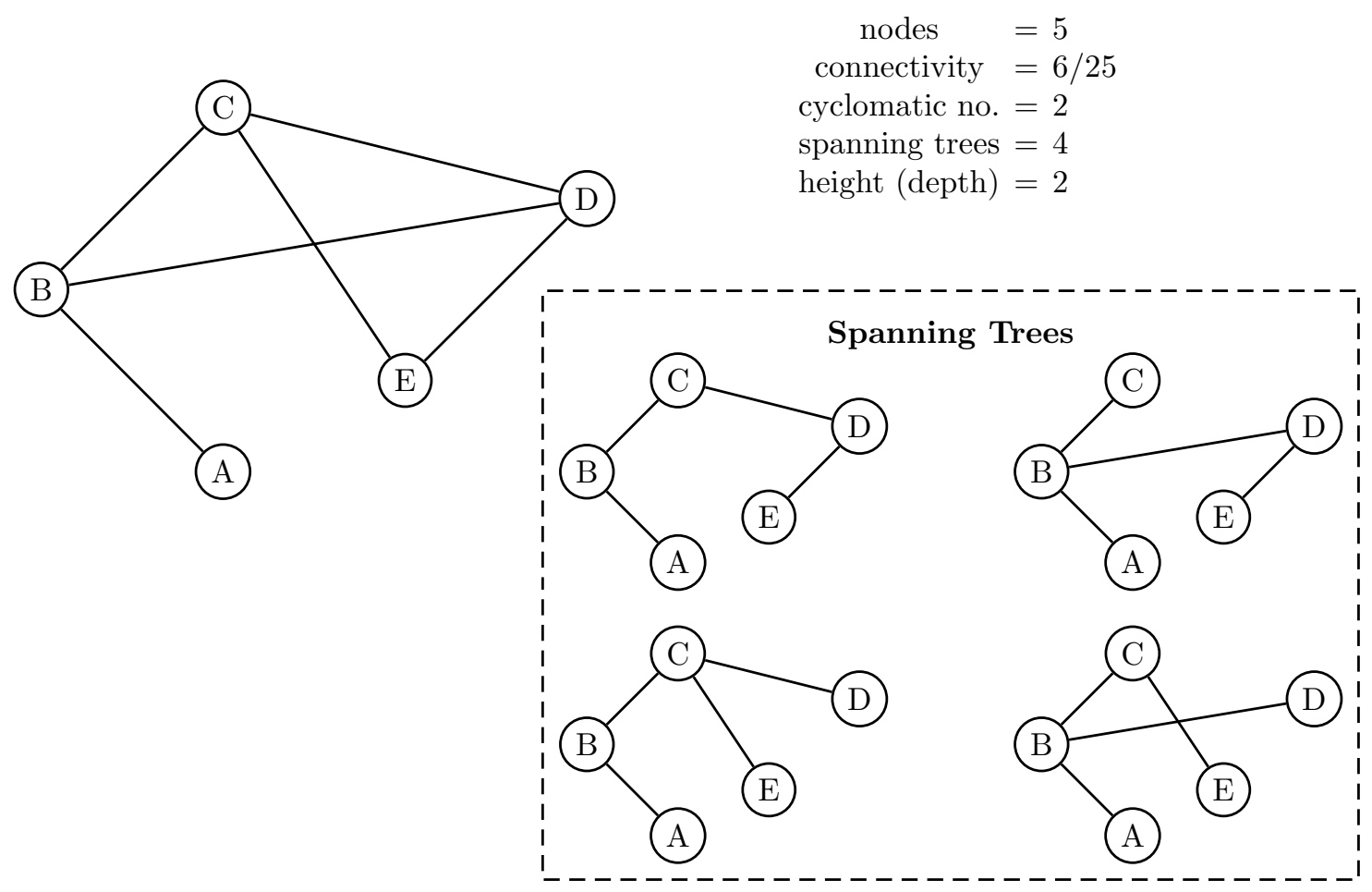

Figure 1: Various graph theoretic measures for a simple graph. The spanning trees are shown in the dashed box

tree. The number of spanning trees increases rapidly with the cyclomatic number.

The height of the flattest spanning tree, or equivalently the maximum number of hops separating two nodes on the graph (popularised in the movie six degrees of separation - which refers to the maximum number of acquaintances connecting any two people in the World) is another useful measure related to complexity. Networks having small degrees of separation (so called small world networks) tend to support more complex dynamics than networks having a large degree of separation. The reason is that any local disturbance is propagated a long way through a small world network before dying out, giving rise to chaotic dynamics, whereas in the other networks, disturbances remain local, leading to simpler linear dynamics.

\subsection{Offdiagonal complexity}

Recently, Claussen (2007) introduced a complexity measure called offdiagonal complexity that is low for regular and randomly connected graphs, but takes on extremal values for scale-free graphs, such as typically seen in naturally occurring networks like metabolic and foodweb networks, the internet, the world wide web and citation networks. The apparent ubiquity of the scale-free property amongst networks we intuitively associate as complex (Newman, 2003) is the justification for using offdiagonal complexity, the other advantage being its computational practicality.

To compute offdiagonal complexity, start with the adjacency matrix

$$
g_{i j}= \begin{cases}1 & \text { if } i \text { and } j \text { are connected } \\ 0 & \text { otherwise }\end{cases}
$$

Let $\ell(i)$ be the node degree of $i$, and let $c_{m n}, m \leq n$, be the number of edges between all nodes $i$ and $j$ with node degrees $m=\ell(i), n=\ell(j)$ :

$$
c_{m n}=\sum_{i, j} g_{i j} \delta_{m, \ell(i)} \delta_{n, \ell(j)}
$$


Then the normalised $n$-th diagonal sum is

$$
a_{n}=\frac{\sum_{i} c_{i, i+n}}{\sum_{i, j} c_{i j}}
$$

The offdiagonal complexity is defined by a Boltzmann-Gibbs entropy-like formula over the normalised diagonal sums:

$$
C_{\text {offdiag }}=-\sum_{n} a_{n} \ln a_{n}
$$

For regular lattices, each node has the same link degree, so $c_{m n}$ is diagonal, $a_{n}=\delta_{n 0}$ and $C_{\text {offdiag }}=0$.

For random graphs, most edges will connect nodes with similar link degree (the characteristic link degree scale), so $c_{m n}$ will have a mostly banded structure, and $a_{n} \rightarrow 0$ as $n$ increases. This leads to small non-zero values of the offdiagonal complexity.

Scale free networks have a power law distribution of link degree, which leads to the $c_{m n}$ matrix having a wide spread of entries. In the case of all $a_{n}$ being equal, offdiagonal complexity takes its maximum value as equal to the number of nodes.

\section{Information as Complexity}

The single simplest unifying concept that covers all of the preceding considerations is information. The more information required to specify a system, the more complex it is. A sandpile is simple, because the only information required is that it is made of sand grains (each considered to be identical, even if they aren't in reality), and the total number of grains in the pile. However, a typical motorcar requires a whole book of blueprints in its specification.

Information theory began in the work of Shannon (1949), who was concerned with the practical problem of ensuring reliable transmission of messages. Every possible message has a certain probability of occurring. The less likely a message is, the more information it imparts to the listener of that message. The precise relationship is given by a logarithm:

$$
I=-\log _{2} p
$$

where $p$ is the probability of the message, and $I$ is the information it contains for the listener. The base of the logarithm determines what units information is measured in - base 2 means the information is expressed in bits. Base 256 could be used to express the result in bytes, and is of course equivalent to dividing equation (5) by 8 .

Shannon, of course, was not so interested in the semantic content of the message (ie its meaning), rather in the task of information transmission so instead considered a message composed of symbols $x_{i}$ drawn from an alphabet $A$. Each symbol had a certain probability $p\left(x_{i}\right)$ of appearing in a message - consider how the letter 'e' is far more probable in English text than the letter ' $\mathrm{q}$ '. These probabilities can be easily measured by examining extant texts. A first order approximation to equation (5) is given by:

$$
I\left(x_{1} x_{2} \ldots x_{n}\right) \approx \sum_{i=1}^{n} p\left(x_{i}\right) \log _{2} p\left(x_{i}\right)
$$

This equation can be refined by considering possible pairs of letters, then possible triplets, in the limit converging on the minimum amount of information required to be transmitted in order for the message to be reconstructed in its original form. That this value may be considerably less that just sending the original message in its entirety is the basis of compression algorithms, such as those employed by the well-known gzip or PKzip (aka WinZip) programs.

The issue of semantic content discouraged a lot of people from applying this formalism to complexity measures. The problem is that a message written in English will mean something to a native English speaker, but be total gibberish to someone brought up in the Amazon jungle with 


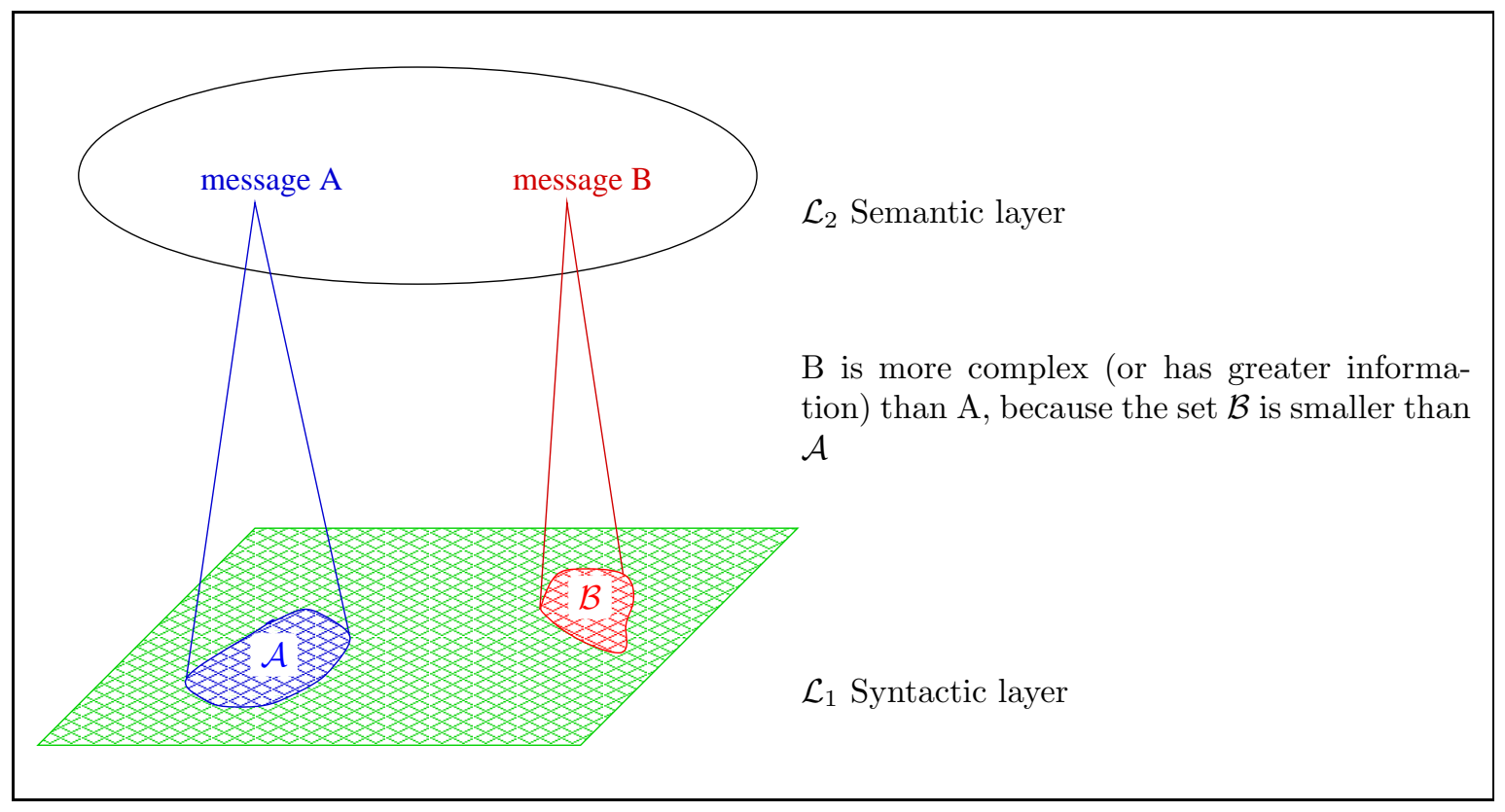

Figure 2: Diagram showing the syntactic and semantic spaces. Two different messages, having meanings $\mathrm{A}$ and $\mathrm{B}$, can each be coded in many equivalent ways in syntactic space, represented by the sets $\mathcal{A}$ and $\mathcal{B}$. The information or complexity of the messages is related to the size it occupies in syntactic space by formula (5)

no contact with the English speaking world. The information content of the message depends on exactly who the listener is! Whilst this context dependence appears to make the whole enterprise hopeless, it is in fact a feature of all of the measures discussed so far. When counting the number of parts in a system, one must make a decision as to what exactly constitutes a part, which is invariably somewhat subjective, and needs to be decided by consensus or convention by the parties involved in the discussion. Think of the problems in trying decide whether a group of animals is one species of two, or which genus they belong to. The same issue arises with the characterisation of the system by a network. When is a relationship considered a graph edge, when often every component is connected to every other part in varying degrees.

However, in many situations, there appears to be an obvious way of partitioning the system, or categorising it. In such a case, where two observers agree on the same way of interpreting a system, then they can agree on the complexity that system has. If there is no agreement on how to perform this categorisation, then complexity is meaningless

To formalise complexity then, assume as given a classifier system that can categorise descriptions into equivalence classes. Clearly, humans are very good at this — they're able to recognise patterns even in almost completely random data. Rorschach plots are random ink plots that are interpreted by viewers as a variety of meaningful images. However, a human classifier system is not the only possibility. Another is the classification of programs executed by a computer by what output they produce. Technically, in these discussions, researchers use a Universal Turing Machine (UTM), an abstract model of a computer.

Consider then the set of possible binary strings, which can fed into a UTM $U$ as a program. Some of these programs cause $U$ to produce some output then halt. Others will continue executing forever. In principle, it is impossible to determine generally if a program will halt or continue on indefinitely. This is the so called halting problem. Now consider a program $p$ that causes the UTM to output a specific string $s$ and then halt. Since the UTM halts after a certain number of instructions executed (denoted $\ell(p)$ ) the same result is produced by feeding in any string starting with the same $\ell(p)$ bits. If the strings have equal chance of being chosen (uniform measure), then the proportion of strings starting with the same initial $\ell(p)$ bits is $2^{-\ell(p)}$. This leads to the 
universal prior distribution over descriptions $s$, also known as the Solomonoff-Levin distribution:

$$
P(s)=\sum_{\{p: U(p)=s\}} 2^{-\ell(p)}
$$

The complexity (or information content) of the description is given by equation (5), or simply the logarithm of (77). In the case of an arbitrary classifier system, the complexity is given by the negative logarithm of the equivalence class size

$$
\mathcal{C}(x)=\lim _{s \rightarrow \infty} s \log _{2} N-\log _{2} \omega(s, x)
$$

where $N$ is the size of the alphabet used to encode the description and $\omega(s, x)$ is the number of equivalent descriptions having meaning $x$ of size $s$ or less (Standish, 2001).

It turns out that the probability $P(s)$ in equation (7) is dominated by the shortest program (Li and Vitányi, 1997, Thm 4.3.3), namely

$$
K(s)+\log _{2} P(s) \leq C
$$

$\left(\log _{2} P(s)<0\right.$ naturally) where $C$ is a constant independent of the description $s . K(s)$ is the length of the shortest program $p$ that causes $U$ to output $s$, and is called the Kolmogorov complexity or algorithmic complexity.

An interesting difference between algorithmic complexity, and the general complexity based on human observers can be seen by considering the case of random strings. Random, as used in algorithmic information theory, means that no shorter algorithm can be found to produce a string than simply saying "print ...", where the ... is a literal representation of the string. The algorithmic complexity of a random string is high, at least as high as the length of the string itself. However, a human observer simply sees a random string as a jumble of letters, much the same as any other random string. In this latter case, the equivalence class of random strings is very large, close to $N^{s}$, so the perceived complexity is small. Thus the human classifier defines an example of what Gell-Mann calls effective complexity (Gell-Mann, 1994), namely a complexity that has a high value for descriptions that are partially compressible by complex schema, but low for random or obviously regular systems.

A good introduction to information theoretical concepts for complex systems studies can be found in (Adami, 1998).

\subsection{Information Theoretic Graph Complexity}

In an attempt to bridge the information theoretic approach to complexity with graph theoretical approaches, Standish recently introduced a coding scheme for which practical (though still NP-hard) algorithms exist for calculating the size of the equivalence class of descriptions (Standish, 2005). The intention is to use this method with networks that have a meaning or function attached, such as metabolic networks, or food webs. A randomly constructed food web will collapse fairly quickly under ecosystem dynamics to a much smaller stable food web, so random or regular networks will tend to have a lower complexity value.

However, when applied to abstract networks, it leads to a perverse result that regular networks have the high complexities, and the completely connected network has maximal complexity. This effect can be ameliorated by introducing a compressed complexity measure, which reduces the complexity measure of regular networks, and is closer to a Turing complete syntactic language. Unfortunately, there is no computationally effective algorithm known for calculating this compressed complexity measure.

\section{Computational Complexity and Logical Depth}

Algorithmic complexity takes no account of the time required to execute the shortest algorithm. An almanac of tide charts and Newton's equations of motion plus law of gravity for the EarthMoon-Sun system contain the same information, yet the almanac contains the information in a 
more useful form for the maritime sailor, as it requires less work to determine when the tides occur. Computational complexity and logical depth are two concepts designed to address the issue of a description's value.

Computational Complexity is defined as the execution time of the algorithm. Since this is highly dependent on what operations are available to the processor, usually only the scaling class of the algorithm is considered as the input size is increased. Algorithms may scale polynomially, which means the execution time increases as some power of the problem size $\left(t \propto n^{s}\right)$, or may scale faster than this (eg exponentially: $t \propto s^{n}$ ), in which case they have nonpolynomial complexity. The class of polynomial algorithms is called $P$, and the class of nonpolynomial algorithms for which the solution can be checked in polynomial time is called $N P$. It is known that all $N P$ algorithms can be transformed into any other by means of a polynomially preprocessor, but it is unknown whether or not $P=N P$, ie whether it is possible to transform any nonpolynomial algorithm into a polynomial one. This issue is of great importance, as certain public key encryption schemes depend on nonpolynomial algorithms to ensure the encryption scheme cannot be cracked within a practical amount of time.

Bennett's logical depth (Bennett, 1988) is the execution time of the most highly compressed representation of an object, relative to some reference machine. It is meant to be a measure of the value of the object - the almanac of tide tables has a high value of logical depth compared with the equations of motion that generate it.

In terms of the observer-based complexity notions introduced in $\$ 4$ assume that the observer has a limited amount of computing resources. Perhaps e is only prepared to spend 5 minutes computing the information, and prior to the widespread availability of electronic computers this meant that the almanac was not equivalent to the equations of motion, since it requires more than 5 minutes to compute the tidal information from the equations of motion via manual paper and pencil techniques. Since the almanac is inequivalent to the equations of motion in this context, it is clear that the almanac has greater complexity in this context.

\section{Occam's Razor}

The practice of preferring a simpler theory over a more complex one when both fit the observed evidence is called Occam's Razor, after William de Occam:

Entities should not be multiplied unnecessarily.

What is not widely appreciated, is that this strategy is remarkably successful at picking better theories. Often, when tested against further empirical evidence, the simpler theory prevails over the more complex. A classical example of this sort of thing is Einstein's General Theory of Relativity. The key field equations of General Relativity are really quite simple:

$$
\mathbf{G}=8 \pi \kappa \mathbf{T}
$$

Of course unraveling what these equations means for a specific instance involves a nontrivial amount of 4 dimensional tensor calculus, so the General Relativity computations have high logical depth. Einstein proposed the equations in this form because they seemed the most "beautiful" - there were a large number of alternative formulations that fitted the data at the time. One by one, these alternative formulations were tested empirically as technology developed through the 20th century, and found wanting.

However, by what criteria is a particular theory more simple than another. Goodman (1972) developed a theory of simplicity to put the practice of Occam's Razor on a more rigorous footing. His idea was to formalise the theories into formal logic predicates, and then count the number of primitive clauses required to encode the theory.

Solomonoff (1964) developed the concept of algorithmic information complexity in the 1960s as a way of explaining why Occam's razor works. He considered the set of all possible descriptions and computed the probability distribution that a particular description would be generated by a 
program picked at random by the reference machine. His work had some technical problems that were solved by Levin (1974), which led to the universal prior distribution (7). Basically, simple descriptions have a much higher probability than more complex ones, thus Occam's razor. The same behaviour is true of the arbitrary classifier system at equation (8) (Standish, 2004).

While the world is expected to be remarkably simple by the above arguments, it is also logically very deep $\left(10^{10}\right.$ years of runtime so far!). This appears to be the result of another poorly understood principle called the Anthropic Principle. The Anthropic Principle states that the world must be consistent with our existence as intelligent, reasoning beings (Barrow and Tipler, 1986). So while Occam's razor says we should live in the simplest of universes, the Anthropic Principle says it shouldn't be too simple, as a certain level of complexity is required for intelligent life. The simplest means of generating this level of complexity is by accruing random mutations, and selecting for functional competence, ie Darwinian evolution.

\section{Complexity as a quality — Emergence}

It is often thought that complex systems are a separate category of systems to simple systems. So what is it that distinguishes a complex system, such as a living organism, or an economy, from a simple system, such as a pair of pliers? This question is related to the notorious question of What is Life?, however may have a simpler answer, since not all complex systems are living, or even associated with living systems.

Consider the concept of emergence (Holland, 1997, Fromm, 2004). We intuitively recognise emergence as patterns arising out of the interactions of the components in a system, but not implicit in the components themselves. Examples include the formation of hurricanes from pressure gradients in the atmosphere, crashes in stock markets, flocking behaviour of many types of animals and of course, life itself.

Let us consider a couple of simple illustrative examples, that are well known and understood. The first is the ideal gas, a model gas made up of large numbers of non-interacting point particles obeying Newton's laws of motion. A thermodynamic description of the gas is obtained by averaging:

temperature $(T)$ is the average kinetic energy of the particles;

pressure $(P)$ is the average force applied to a unit area of the boundary by the particles colliding with it;

density $(\rho)$ is the average mass of particles in a unit volume;

The ideal gas law is simply a reflection of the underlying laws of motion, averaged over all the particles:

$$
P \rho \propto T
$$

The thermodynamic state is characterised by the two parameters $T$ and $\rho$. The so-called first law of thermodynamics is simply a statement of conservation of energy and matter, in average form.

An entirely different quantity enters the picture in the form of entropy. Consider discretising the underlying phase-space into cubes of size $h^{N},(N$ being the number of particles $)$ and then counting the number of such cubes having temperature $T$ and density $\rho, \omega(T, \rho, N)$. The entropy of the system is given by

$$
S(T, \rho, N)=k_{B} \ln \omega(T, \rho, N)
$$

where $k_{B}$ is a conversion constant that expresses entropy in units of Joules per Kelvin. One can immediately see the connection between complexity (eq. 8) and entropy. Readers familiar with quantum mechanics will recognise $h$ as being an analogue of Planck's constant. However, the ideal gas is not a quantum system, and as $h \rightarrow 0$, entropy diverges! However, it turns out that in the thermodynamic limit $(N \rightarrow \infty)$, the average entropy $S / N$ is independent of the size of $h$.

The second law of thermodynamics is a recognition of the fact that the system is more likely to move a state occupying a larger region of phase space, than a smaller region of phase space, namely that $\omega(T, \rho, N)$ must increase in time. Correspondingly entropy must also increase (or 
remain constant) over time. This is a probabilistic statement that only becomes exact in the thermodynamic limit. At the syntactic, or specification level of description (ie Newton's laws of motion), the system is perfectly reversible (we can recover the system's initial state by merely reversing the velocities of all the particles), yet at the semantic (thermodynamic) level, the system is irreversible (entropy can only increase, never decrease).

The property of irreversibility is an emergent property of the ideal gas, as it is not entailed by the underlying specification. It comes about because of the additional identification of thermodynamic states, namely the set of all micro-states possessing the same temperature and density. This is extra information, which in turn entails the second law.

The second example I'd like to raise (but not analyse in such great depth) is the well known Game of Life, introduced by John Conway (1982). This is a cellular automaton (Wolfram, 1984), in this case, a 2D grid of cells where each cell can be one of two states. Dynamics on the system is imposed by the rule that the state of a cell depends on the values of its immediate neighbours at the previous time step.

Upon running the Game of Life, one immediately recognises a huge bestiary of emergent objects, such as blocks, blinkers and gliders. Take gliders for example. This is a pattern that moves diagonally through the grid. The human observer recognises this pattern, and can use it to predict the behaviour of the system with less effort than simulating the full cellular automaton. It is a model of the system. However, the concept of a glider is not entailed by the cellular automaton specification, which contains only states and transition rules. It requires the additional identification of a pattern by the observer.

This leads to a general formulation of emergence (Standish, 2001). Consider a system specified in a language $\mathcal{L}_{1}$, which can be called the specification, or syntactic layer (see figure 21). If one accepts the principle of reduction, all systems can ultimately be specified the common language of the theoretical physics of elementary particles. However, an often believed corollary of reduction is that this specification encodes all there is to know about the system. The above two examples shows this corollary to be manifestly false. Many systems exhibit one or more good models, in another language $\mathcal{L}_{2}$, which can be called the semantic layer. The system's specification does not entail completely the behaviour of the semantic model, since the latter also depends on specific identifications made by the observer. In such a case, we say that properties of the semantic model is emergent with respect to the syntactic specification.

The concept of "good" model deserves further discussion. In our previous two examples, neither the thermodynamic model, nor the glider model can be said to perfectly capture the behaviour of the system. For example, the second law of thermodynamics only holds in the thermodynamic limit - entropy may occasionally decrease in finite sized systems. A model based on gliders cannot predict what happens when two gliders collide. However, in both of these cases, the semantic model is cheap to evaluate, relative to simulating the full system specification. This makes the model "good" or "useful" to the observer. We don't prescribe here exactly how to generate good models here, but simply note that in all cases of recognised emergence, the observer has defined a least one semantic and one syntactic model of the system, and that these models are fundamentally incommensurate. Systems exhibiting emergence in this precise sense can be called complex.

A school of thought founded by Robert Rosen holds that complex systems cannot be described by a single best model as reductionists would assume, but rather has a whole collection of models that in the limit collectively describe the system (Rosen, 1991). That such systems exist, at least formally, is assured by Gödel's incompleteness theorem, (Hofstadter, 1979) which shows that number theory is just such a system that cannot be captured by a finite specification. He further argues mechanical systems (those that have a finite specification such as the examples I have given above) can never be complex, since the specification contains all there is to know about the system. However, he implicitly assumes that all models must be perfect (ie in perfect correspondence with the underlying system), rather than merely good as I do here. This constitutes a straw man argument, and leads him to the false conclusion that mechanical systems (eg computer simulations) can never exhibit emergence. The two examples presented above, which are perfectly good mechanical systems, are counter-examples to this claim. Furthermore, the definition of complex systems presented here is known to be non-empty, a fact not known of Rosen's definition 
since no physical counterpart to Gödel's incompleteness theorem is known.

\section{Conclusion}

When connoting a quality, complexity of a system refers to the presence of emergence in the system, or the exhibition of behaviour not specified in the systems specification. When connoting a quality, complexity refers to the amount of information needed to specify the system. Both notions are inherently observer or context dependent, which has lead to a disparate collection of formalisations for the term, and has lead to some despairing of the concept being adequately formalised. This would be a mistake, as within a given application, the meaning can be well defined.

An additional difficulty is the combinatorial size of the underlying syntactic space, which can lead to the intractability of computing complexity. Furthermore, the details of the syntactic layer may be inaccessible, for example the absence of historical genetic data in the study of evolution from the fossil record. So being able to establish easy to measure proxies for complexity is often important, and many proposals for complexity are of this nature.

\section{Bibliography}

\section{References}

Adami, C. (1998). Introduction to Artificial Life. Springer.

Albert, R. and Barabási, A.-L. (2002). Statistical mechanics of complex networks. Reviews of Modern Physics, 74:47.

Barrow, J. D. and Tipler, F. J. (1986). The Anthropic Cosmological Principle. Clarendon, Oxford.

Bennett, C. H. (1988). Logical depth and physical complexity. In Harkin, R., editor, The Universal Turing Machine: A Half Century Survey, volume 1, pages 227-258. Oxford UP.

Bonner, J. T. (1988). The Evolution of Complexity. Princeton UP, Princeton.

Claussen, J. C. (2007). Offdiagonal complexity: A computationally quick complexity measure for graphs and networks. Physica A, pages 365-373. arXiv:q-bio.MN/0410024.

Conway, J. H. (1982). What is life? In Berlekamp, E., Conway, J. H., and Guy, R., editors, Winning Ways for your Mathematical Plays, volume 2, chapter 25. Academic, New York.

Diestel, R. (2005). Graph Theory. Springer, Berlin, 3rd edition.

Edmonds, B. (1999). Syntactic Measures of Complexity. PhD thesis, University of Manchester. http://bruce.edmonds.name/thesis/, Complexity Bibliography: http://bruce.edmonds.name/compbib/.

Erdös, P. and Rényi, A. (1959). On random graphs. Publ. Math. Dubrecen, 6:290-291.

Fromm, J. (2004). The Emergence of Complexity. Kassel UP.

Gell-Mann, M. (1994). The Quark and the Jaguar: Adventures in the Simple and the Complex. Freeman.

Goodman, N. (1972). Problems and Projects. Bobbs-Merrill, Indianapolis.

Hofstadter, D. R. (1979). Gödel, Escher, Bach: an Eternal Golden Braid. Harvester.

Holland, J. (1997). Emergence: From Chaos to Order. Addison Wesley. 
Levin, L. A. (1974). Laws of information conservation (non-growth) and aspects of the foundation of probability theory. Problems Inform. Transmission, 10:206-210.

Li, M. and Vitányi, P. (1997). An Introduction to Kolmogorov Complexity and its Applications. Springer, New York, 2nd edition.

McShea, D. W. (1996). Metazoan complexity and evolution: Is there a trend? Evolution, 50:477492.

Newman, M. E. J. (2003). The structure and function of complex networks. SIAM Review, 45(2):167-256. arXiv:cond-mat/0303516.

Rosen, R. (1991). Life Itself. Columbia UP, New York.

Shannon, C. E. (1949). The Mathematical Theory of Communication. Urbana.

Solomonoff, R. J. (1964). A formal theory of inductive inference, part 1 and 2. Inform. Contr., pages $1-22,224-254$.

Standish, R. K. (2001). On complexity and emergence. Complexity International, 9. arXiv:nlin.AO/0101006.

Standish, R. K. (2004). Why Occam's razor? Foundations of Physics Letters, 17:255-266. arXiv:physics/0001020.

Standish, R. K. (2005). Complexity of networks. In Abbass et al., editors, Recent Advances in Artificial Life, volume 3 of Advances in Natural Computation, pages 253-263, Singapore. World Scientific. arXiv:cs.IT/0508075.

Watts, D. J. and Strogatz, S. H. (1998). Collective dynamics of 'small-world' networks. Nature, 393(6684):409-10.

Wolfram, S. (1984). Cellular automata as models of complexity. Nature, 311:419-424.

\section{Glossary}

Algorithmic Complexity Length of shortest program capable of generating the description (also known as Kolmogorov complexity)

Anthropic Principle The statement that the properties of the universe must be such as to permit our existence as human observers

Cellular Automaton A grid of cells, each of which can be in a finite number of states. The states of each cell depend only on the states of a neighbourhood of cells at the previous timestep, known as a transition rule.

Church-Turing Thesis The proposition that all computable functions can be computed on a Turing machine.

Classifier system A system that classifies a set of objects into a discrete set of classes. Formally equivalent to a map into the set of whole numbers.

Complexity (quality) The quality of possessing emergent properties

Complexity (quantity) The amount of information a particular system represents to the observer

Computational Complexity The computational cost of executing an algorithm.

Emergence The phenomenon of emergent properties 
Emergent Properties Properties of a system at the semantic level that are not entailed at the syntactic level

Entail To logically imply something

Entropy Logarithm of number of syntactic states equivalent to a given semantic state. It is related to information $(I)$ by $S+I=S_{\max }$ where $S_{\max }$ is the maximum possible entropy of the system.

Equivalence Class A set of objects that are equivalent under some mapping i.e. $\{x: e(x)=$ $\left.e\left(x^{\prime}\right)\right\} \exists x^{\prime}$

Game of Life A well known cellular automaton, with two states per cell, and a particular transition rule

Graph Theory Mathematical theory of objects consisting of atomic nodes linked by connections called edges

Gödel Incompleteness Theorem No finite set of axioms can prove all possible true theorems of number theory

Information The amount of meaning in any description; formally given as the logarithm of the proportion of syntactic space occupied by the description

Link degree the number of edges a node has in a graph

Logical Depth Execution time of the most compressed representation of an object

Newton's Laws of motion Laws of ideal point particles: the acceleration a particle experiences is proportional to the force acting on it, which is a function of the positions and velocities of the particle and the environment

Occam's Razor A statement of the practice of preferring a simpler theory over a more complex one

Scale free A scale free distribution has infinite mean. A power law distribution is a common scale free distribution. A scale free process is a stochastic process obeying a scale free distribution.

Second Law of Thermodynamics Entropy can only increase, or remain constant in a closed system; it can never decrease

Semantic Level (or space) The space of meanings for any description.

Syntactic Level (or space) The language in which a description is specified: letters of the alphabet, genetic code, laws of theoretical physics, as appropriate

Turing Machine A formal model of a computation.

Universal Turing Machine A formal model of a computer. Is capable is simulating any Turing Machine with appropriate input. 